\title{
Standardization of laparoscopically assisted vaginal hysterectomy for uterine myoma and uterine adenomyosis at a single institution
}

\author{
近畿大学医学部
}

岡田紀久子、塩田 充、小谷泰史、梅本雅彦、飛梅孝子、宇賀神奈月、星合 昊

\section{Standardization of laparoscopically assisted vaginal hysterectomy for uterine myoma and uterine adenomyosis at a single institution}

Kikuko Okada MD, Mitsuru Shiota MD, Yasushi Kotani MD, Masahiko Umemoto MD, Takako Tobiume MD, Natsuki Ugajin MD, Hiroshi Hoshiai MD

Department of Obstetrics and Gynecology, Kinki University Faculty of Medicine

\begin{abstract}
Objective: Total vaginal hysterectomy (TVH) and total abdominal hysterectomy (TAH) are presently the standard procedures for total hysterectomy. Since the advent of laparoscopically assisted vaginal hysterectomy (LAVH), our institution has actively performed LAVH to establish it as standardized procedure for patients with uterine myomas and those with uterine adenomyosis. This report investigates the parameters to determine LAVH to be considered a standardized procedure.

Method: In our institution, 282 LAVH cases were performed from 1995 to 2001 (Group I), 258 cases from 2002 to 2010 (Group IIa) by "experts", and 83 cases by non-expert Ob/Gyn surgeon (Group IIb). The groups were compared by age and parity of the patients; uterine weight; operative time; blood loss; rates of intraoperative conversion to open surgery; and intra- and post-operative complications. The trend in the annual rate of LAVH was also examined.

Results: There were no significant differences in age, parity, blood loss, or conversion to open surgery. Uterine weight was significantly lower $(350 \mathrm{~g})$ and operative time significantly longer (163min) in Group IIb than in Groups I $(400 \mathrm{~g} / 143 \mathrm{~min})$ and IIa $(411 \mathrm{~g} / 143 \mathrm{~min})$. The annual LAVH rate has increased continually, from $16.2 \%$ in 1995 to $85.7 \%$ in 2010.

Discussion: Because no difference was observed in blood loss or complication rates among the three groups of patients, the authors have concluded that LAVH can be performed by all gynecological surgeons. Presently, LAVH is performed for the majority of patients requiring total hysterectomy. Therefore, LAVH has been established as the institution's standardized procedure for total hysterectomy.
\end{abstract}

Key Words: Total hysterectomy, laparoscopically assisted vaginal hysterectomy, standardization

\section{Objective}

Uterine myoma is a benign tumor that develops in the muscle layer of the uterus. Myomata are estimated to occur in as many as $20 \%$ of women at the age of 30 and above, and $40 \%$ of women at the age of 40 and above ${ }^{1,2)}$. Uterine adenomyosis is characterized by ectopic spread of the endometrium into the myometrium, which is commonly reported to have a frequency of about $20 \%$. Both of these conditions can cause menorrhagia and anemia, and total hysterectomy may be performed as a treatment for patients with severe symptoms. The conventional approaches to total hysterectomy have been the total vaginal 
hysterectomy (TVH) and the total abdominal hysterectomy (TAH). In TVH, all of the surgical steps are performed transvaginally and the uterus is removed through the vagina, causing less operative trauma compared with TAH, which requires laparotomy. However, the indication for TVH is limited by tumor size, elasticity of the vagina, past surgical history and other considerations. Until recently, in cases where TVH is contraindicated, TAH has been selected. Both TVH and TAH have a long history. They have been performed since the $18^{\text {th }}$ century, and both procedures have been established as the standard operative methods for total hysterectomy. Into these circumstances, only twenty years ago, the laparoscopic hysterectomy was introduced ${ }^{3)}$. Several laparoscopic approaches to hysterectomy, including the laparoscopically assisted vaginal hysterectomy, have since developed.

Since 1995, when laparoscopically assisted vaginal hysterectomy (LAVH) was initiated in our institution, we have actively sought to replace highly invasive TAH with vaginal surgery. Our final goal is to establish a safe and universal method of LAVH as a standard operative procedure.

As with TVH, indications for LAVH are limited by tumor size and other considerations ${ }^{4)}$. Varying contraindications to LAVH based on tumor size have been reported in different studies depending on the experience and skill of the operating surgeons ${ }^{56)}$. In these studies, the maximum tumor size for LAVH was established based on the initial results of two operating surgeons who were certified in endoscopic techniques. These experts found that the average blood loss for LAVH compared with other operative methods was significantly higher when the weight of the removed uterus was greater than or equal to $800 \mathrm{~g}$. Thus, the indication that the uterus should be no heavier than 800 g (i.e. size consistent with approximately 16 weeks' gestation on pelvic exam) for LAVH was established ${ }^{7)}$, and since, LAVH was performed by the other $\mathrm{Ob} / \mathrm{Gyn}$ surgeons at the institution, and the indication has been confirmed in a follow-up study ${ }^{6)}$.

Standardization of surgery here signifies the process of establishing a surgical procedure that should become the standard. We believe the following conditions are required: 1 ) the results of the procedure are equivalent to or superior to those of other standard surgeries; 2) the procedure is safe and universal; and 3) the procedure is performed as often as other standard surgeries. The process of standardization begins with the establishment of the procedure, followed by the establishment of the indication, followed by the standardization. Standardization can be institution-wide, specialty-wide, nation-wide, etc.

An extensive literature search failed to uncover any reports regarding the institutional establishment of a standardized operative method for total hysterectomy. The current study has evaluated whether LAVH has been established as a standardized operative method for patients with uterine myomas and those with uterine adenomyosis in our institution.

\section{Patients and Methods}

A total of 669 patients were indicated for LAVH during the study period. Among them, 600 patients who actually underwent LAVH were included in this study. Table 1 summarizes the criteria for LAVH and TVH candidates. In principle, TAH is performed for uteri larger than those indicated for LAVH or TVH. The patients were classified into two groups; 282 patients who underwent surgery from January 1995 to December 2001 by the two experts (Group I), and 387 patients who underwent surgery from January 2002, after the operative procedure and the indications for LAVH were established, to December 2010 (Group II). Group II was divided into two subgroups: 258 patients who were operated by one of 7 experts (surgeons who have been certified in endoscopic techniques by Japan Society of Obstetrics and Gynecology; Group IIa) and 83 patients who were operated by one of 12 other $\mathrm{Ob} / \mathrm{Gyn}$ surgeons at the institution under the supervision of an expert (Group IIb). Group IIb comprised 
Table 1. Criteria for TVH and LAVH candidates

Criteria for candidates for total vaginal hysterectomy

\begin{tabular}{|c|}
\hline History of vaginal delivery \\
\hline Uterine size smaller than a fist \\
\hline No history of laparotomy \\
\hline (excluding appendectomy) \\
\hline No clear complications of an ovarian tumor \\
\hline No complications of advanced endometriosis \\
\hline
\end{tabular}

Criteria for candidates for laparoscopically-assisted vaginal hysterectomy

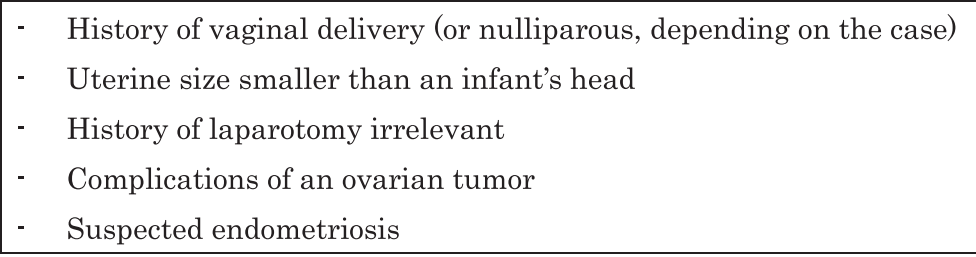

Table 2 Definitions of intra- and post-operative complications

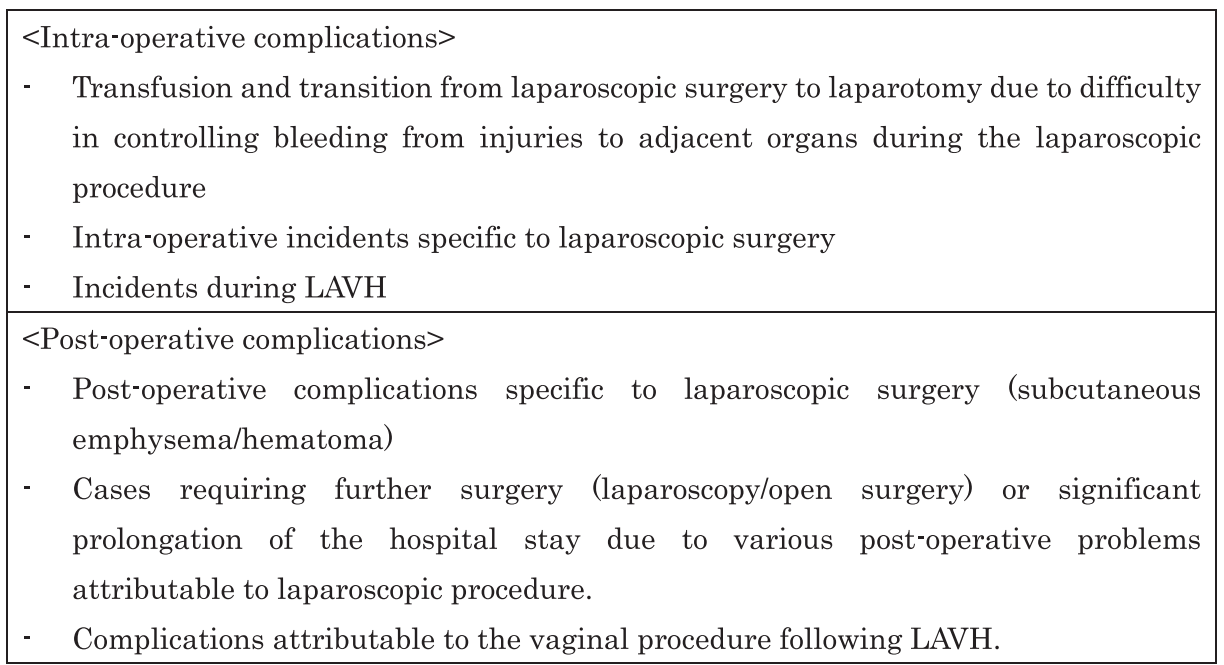

mainly the patients with postoperative uterine weight $<400 \mathrm{~g}$ allocated to the group on the basis of preoperative assessment of uterine size and mobility by expert surgeons. Comparative analysis was performed among these three groups based on patient background (age and parity), surgical details (weight of the removed uterus, operative duration, and blood loss during surgery), the rates of conversion to laparotomy due to intraoperative complications, and rates of intra- and post-operative complications (see definitions in Table 2). The data of the 69 cases in which LAVH was converted to laparotomy were included only in the analysis of complications. In addition, the number and percentage of LAVH among the total number of hysterectomies performed at the institution was evaluated by fiscal year from 1995 to 2010 .

\section{Surgical Procedure}

Pneumoperitoneum is created by a closed method placing the pneumoperitoneum needle through the umbilicus and inserting a uterine manipulator. After the pneumoperitoneum is established, a trocar is inserted through the umbilicus through which the laparoscope is introduced. The second trocar is placed in the midline of the lower abdomen with the third and fourth trocars to its right and left. The size of all the trocars used for the surgery is $5 \mathrm{~mm}$. Under laparoscopic guidance, the Pouch of Douglas and the course of the ureters are 
identified and the round ligaments are coagulated and divided. The fallopian tubes are then coagulated and divided, followed by the proper ovarian ligaments. The peritoneum of the vesico-uterine pouch is opened, and the following procedures are performed vaginally: 1) after retracting the bladder and opening the pouch of Douglas, the uterosacral ligament is divided and ligated. 2) After dividing and ligating the base of the cardinal ligaments and opening the vesicouterine pouch, the uterine arteries are ligated and divided and the uterus is removed. 3) After ensuring hemostasis, the vaginal wall is sutured. The procedure is then completed under laparoscopic guidance. Hemostasis is confirmed again and the cut ends of the uterosacral ligaments are drawn into approximation and sutured to the vaginal cuff. The surgery is completed with peritoneal lavage.

For cases requiring adhesiolysis or those requiring adnexectomy, the same procedure is performed after lysis of adhesions or adnexectomy

\section{Statistical Analysis}

In statistical analysis, ANOVA was used and Scheffe's test was performed for comparisons among the three groups. Chi-square test was used for the comparison of percentages. For all the tests, $\mathrm{p}$ value $<0.05$ was considered statistically significant. StatFlex Version 6.0 was used for analysis.

\section{Results}

Table 3 shows the comparisons of surgical result parameters among Groups I, IIa, and IIb. No significant difference was observed in the

Table 3 Comparisons of parameters among Groups I, Ila, and Ilb

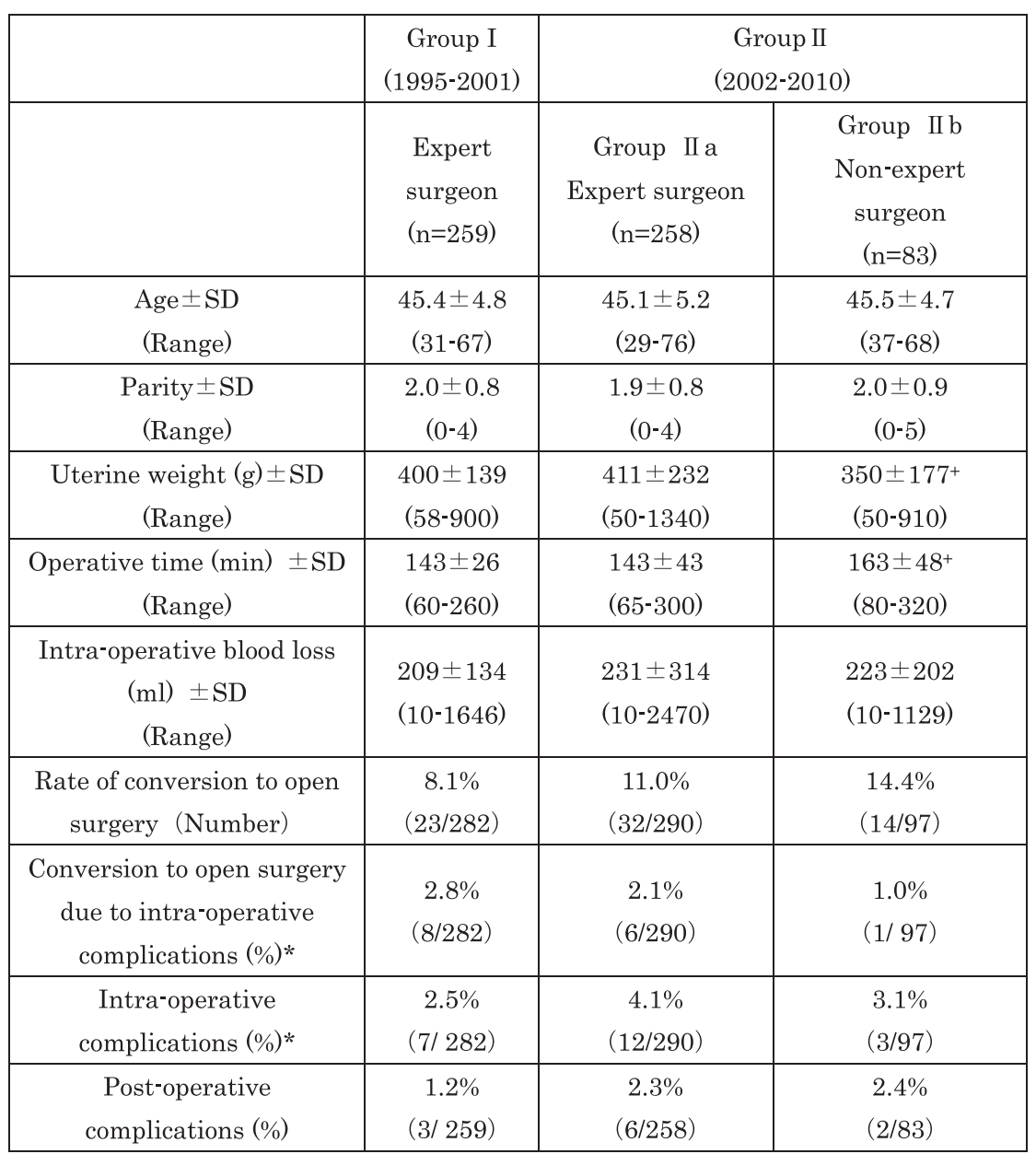

Note: Cases converted to open surgery are included in *

${ }^{+} \mathrm{p}<0.05$ when compared with the other groups 
Table 4 Breakdown of annual total hysterectomy cases by method

\begin{tabular}{|c|c|c|c|c|c|c|c|}
\hline Fiscal year & Total & TAH $^{*}$ & LAVH & LAVH $^{* *}$ & TVH & TLH & LSH \\
\hline 1995 & 136 & $97(71.3) \%$ & $22(16.2) \%$ & 0 & $17(12.5)^{\%}$ & 0 & 0 \\
\hline 1996 & 124 & $62(50.0)$ & $43(34.7)$ & 0 & $19(15.3)$ & 0 & 0 \\
\hline 1997 & 111 & $48(43.2)$ & $52(46.8)$ & 0 & $11(9.9)$ & 0 & 0 \\
\hline 1998 & 83 & $29(34.1)$ & $42(50.6)$ & 0 & 12(14.5) & 0 & 0 \\
\hline 1999 & 98 & $22(22.4)$ & $59(60.2)$ & $6(6.1) \%$ & $11(11.2)$ & 0 & 0 \\
\hline 2000 & 75 & $19(25.3)$ & $49(65.3)$ & 1(1.3) & $6(8.0)$ & 0 & 0 \\
\hline 2001 & 72 & $24(33.3)$ & $42(58.3)$ & $3(4.2)$ & $3(4.2)$ & 0 & 0 \\
\hline 2002 & 55 & $14(25.5)$ & $38(69.1)$ & 1(1.8) & 2(3.6) & 0 & 0 \\
\hline 2003 & 69 & $23(33.3)$ & $40(58.0)$ & $1(1.5)$ & $5(7.3)$ & 0 & 0 \\
\hline 2004 & 66 & $24(36.4)$ & $34(51.5)$ & $4(6.1)$ & $4(6.1)$ & 0 & 0 \\
\hline 2005 & 60 & $19(31.7)$ & $38(63.3)$ & 0 & $3(5.0)$ & 0 & 0 \\
\hline 2006 & 61 & 19(31.1) & $41(67.2)$ & 0 & 1(1.6) & 0 & 0 \\
\hline 2007 & 60 & $19(31.7)$ & $39(65.0)$ & 1(1.6) & 0 & $1(1.6) \%$ & 0 \\
\hline 2008 & 58 & $13(22.4)$ & $43(74.1)$ & 0 & 0 & 1(1.7) & $1(1.7) \%$ \\
\hline 2009 & 49 & $11(22.4)$ & $32(65.3)$ & 0 & 0 & $2(4.1)$ & $4(8.2)$ \\
\hline 2010 & 42 & 5(11.9) & $36(85.7)$ & 0 & 0 & $1(2.4)$ & 0 \\
\hline
\end{tabular}

average age of the patients (45.4 years, 45.1 years, and 45.5 years for Groups I, IIa, and IIb, respectively) or in parity (2.0, 1.9, and 2.0). The uterine weight was significantly lower in the patients in Group IIb compared with other groups $(400 \mathrm{~g}, 411 \mathrm{~g}$, and $350 \mathrm{~g}$, I, IIa, IIb). Operative time was significantly longer in Group IIb than in the other groups (143 minutes, 143 minutes, and 163 minutes, I, IIa, IIb). There was no difference in blood loss $(209 \mathrm{ml}, 231 \mathrm{ml}$, and $223 \mathrm{ml})$, rate of conversion to laparotomy $(8.1 \%$, $11.0 \%$, and $14.4 \%$ ), rate of conversion to laparotomy due to intraoperative complications $(2.8 \%, 2.1 \%$, and $1.0 \%)$, or rate of intraoperative and postoperative complications $(2.5 \%, 4.1 \%$, and $3.1 \% ; 1.2 \%, 2.3 \%$, and $2.4 \%)$. Table 4 shows the number and percentage of patients who underwent LAVH among the total number of patients who underwent total hysterectomy by fiscal year. The rate of LAVH, which was $16.2 \%$ in 1995 , exceeded $50 \%$ by 1998 and has increased steadily since then, reaching $85.7 \%$ in 2010.

\section{Discussion}

The advent of the laparoscopic hysterectomy has allowed the highly invasive TAH to be replaced in many cases with less invasive laparoscopic and laparoscopically assisted procedures ${ }^{8.9}$. The institution in the current study has actively performed LAVH beginning since 1995, led by two experts, whose work established that the procedure could be safely performed in patients with uterine weights of less than $800 \mathrm{~g}^{7)}$. According to a study by Tobiume et al, uterine weight of $800 \mathrm{~g}$ is regarded as equivalent to a diameter of approximately $12 \mathrm{~cm}$, or uterine size consistent with about 16 weeks' gestation on pelvic examination ${ }^{10)}$. Since the establishment of these guidelines in 2002, LAVH has been performed by all of the $\mathrm{Ob} / \mathrm{Gyn}$ surgeons in this hospital. The Japan Society of Obstetrics and Gynecology started an endoscopic surgery certification program in 2003. Seven certified experts (including the two who had already been otherwise certified before 2001) and 12 other $\mathrm{Ob} / \mathrm{Gyn}$ surgeons now perform LAVH at the institution in this study. During the study period, the average uterine weights were lower and the average operative time was longer in cases performed by the surgeons who had not 
been certified (Group IIb). Surgeons with less expertise in laparoscopic procedures tend to choose less difficult cases initially, and require longer operative times because the procedure involves didactic components. However, no difference was observed between any groups in the surgically critical factors of blood loss and complication rates. Thus, the authors have concluded that LAVH is an operative procedure that can be safely performed by all $\mathrm{Ob} / \mathrm{Gyn}$ surgeons, including non-expert surgeons working under the supervision of an expert.

The surgeon allocation procedure in our institution is as follows. First, an expert endoscopic surgeon assesses preoperative uterus size and mobility to determine whether the case should be allocated to a non-expert surgeon. A uterine weight $<400 \mathrm{~g}$ is expected to ensure sufficient visual field to divide the blood vessels and ligaments and to perform the resection treatment that follows, while a uterine weight from 400-800g will not ensure a good visual field. Therefore, non-exert surgeons should perform LAVH only for cases with uterine weight $<400 \mathrm{~g}$.

It is customary for residents at our institution to rotate among 4 sections: tumor, reproductive endocrinology, perinatal, and endoscopy. Rotations are on a 3-month basis, allowing residents to participate in the endoscopic section more than 3 times before completing their residency. During this time, they can be trained in the basics of LAVH and other laparoscopic procedures, including treatment of ovarian cysts and ectopic pregnancies. Residents who wish to specialize in endoscopy will be trained for more challenging procedures, including total laparoscopic hysterectomy (TLH), LM, and treatment of malignancy.

The percentage of patients who have undergone total hysterectomy by LAVH has increased continuously every year, and the procedure is currently the predominant technique for total hysterectomy in the institution where the present study was performed. LAVH has become the mainstay of surgical procedures for total hysterectomy in this institution.

Although limitations to its indications exist, LAVH has now become a standard surgical procedure at the hospital presented in this report. It is considered a safe and universal operative technique that can be performed by any surgeon for patients with uteri smaller than $12 \mathrm{~cm}$ (a size consistent with a uterus at 16 weeks' gestation on pelvic exam).

TVH that does not involve laparoscopic assistance may be considered less invasive than LAVH. However, our past study showed that CRP values and WBC counts were significantly lower in LAVH than in TAH and TVH, and the complication rates in LAVH were comparable to those in the other procedures ${ }^{11)}$. Therefore, we recommend primary consideration of LAVH, which is less intrusive than TAH and TVH, and can be performed for nulliparous and relatively large uteri, up to the indicated limits described above.

The total laparoscopic hysterectomy (TLH) is another laparoscopic hysterectomy procedure that has recently been introduced. Shiota et al recently described a survey conducted in Japan that showed that TLH technique has overtaken LAVH as the procedure of choice for laparoscopically assisted total hysterectomy ${ }^{12)}$. Although TLH eliminates the necessity for vaginal manipulation, it requires greater endoscopic skill because the entire procedure is performed laparoscopically. TLH has been found to result in less blood loss than $\mathrm{LAVH}^{13)}$, but some authors have reported that TLH is associated with longer operative times or a higher incidence of urinary tract complications $^{14,15)}$. Now that LAVH has been established as a standardized surgical method in the hospital presented in this study, future goals will include greater reductions in blood loss and fewer complications. More difficult laparoscopic procedures, such as TLH should also be more actively performed. However, academic facilities such as the one in this study must continue to ensure that gynecologic surgeons become proficient in vaginal procedures. Thus, the institutional standardization of LAVH is also 
required for the purpose of satisfactory education.

\section{Conflicts of Interest}

All authors have no conflict of interest in this study.

\section{References}

1) Henderrickson ME, Kempson RL.: Surgical Pathology of the Uterine Corpus. (ed. WB Saunders), 1980; Philadelphia.

2) Parsons L, Sommers SC.: Gynecology, 2nd ed. (ed. WB Saunders), 1978; Philadelphia.

3 ) Reich H.: Laparoscopic hysterectomy. Journal of Gynecolgic Surgery 1989; 5: 213-216.

4) Chapron C, et al: Hysterctomy techniques used for benign pathologies: results of a French multicenter study. Hum Repro 1999; 14: 2464-2470.

5 ) Marana R, et al.: Laparoscopically assisted vaginal hysterectomy versus total hysterectomy: a prospective, randomized, multicenter study. Am J Obstet Gynecol 1999; 180: 270-275.

6 ) Shiota M, et al.: Indication for laparoscopicallyassisted vaginal hysterectomy. JSLS 2011; 15: 343345.

7 ) Shiota M, et al:: The actual status and evalutation of laparoscopically assisted vaginal hysterectomy (LAVH) . Obstetrical and Gynecological Therapy 2003: 86; 307-311. [Article in Japanese]

8 ) Ottosen C, et al.: Three methods for hysterectomy: a randomized, prospective study of short term outcome. BJOG 2000; 107: 1380-1385.

9 ) Seracchioli R, et al:: Total laparoscopic hysterectomy compared with abdominal hysterectomy in the presence of a large uterus. J Am Assoc Gynecol Laparosc 2002; 9: 333-338.

10) Tobiume T, et al.: A study on preoperative estimation of uterine weight in patients with uterine myoma. Japanese Journal of Gynecologic and Obstetric Endoscopy 2008; 24: 213-217. [Article in Japanese]

11) Shiota M, et al. Total Abdominal Hysterectomy versus Laparoscopically-assisted Vaginal Hysterectomy versus Total Vaginal Hysterectomy Comparisons of patient parameters and operative results by method. Asian J Endosc Surg. 2011; 4: 161-5.

12) Shiota M, et al: National investigation on the rates of open surgery and laparoscopic surgery (in fiscal year 2008). Gynecologic and Obstetric Surgery 2010, 21; 127-131. [Article in Japanese]

13) Twijnstra AR, et al.: Twenty-first century laparoscopic hysterectomy: should we not leave the vaginal step out? Gynecol Surg 2009; 6: 311-316.

14) Johnson N, et al:: Surgical approach to hysterectomy for benign gynaecological disease. Cochrane Database Syst Rev. (1): CD003677, 2005.

15) Nieboer TE, et al.: Surgical approach to hysterectomy for benign gynaecological disease. Cochrane Database Syst Rev. (3): CD003677, 2009. 